\title{
High-performance Control \\ for a Nonlinear Biotechnological Process \\ Based-on Adaptive Gain Sliding Mode Strategy
}

\author{
Semcheddine Bouyahia*, Samia Semcheddine, \\ Billel Talbi, Oussama Boutalbi
}

\author{
Department of Electronics \\ University of Ferhat Abbas Sétif 1 \\ Sétif 19000, Algeria \\ E-mails: adelbouyahia@gmail.com, tssamia@yaho.fr, \\ bilel_ei@live.fr, botalbioussama@gmail.com
}

*Corresponding author

Received: June 08, 2018

Accepted: March 26, 2020

Published: June 30, 2020

\begin{abstract}
The production of biogas in anaerobic fermentation is one of the most important interests in industrial applications for two main reasons: the elimination of organic waste and the production of renewable energy. The mentioned biotechnological process, which takes place inside of a continuous stirred tank bioreactor (CSTB), is described in this study by a two-step reaction scheme based on a second-order nonlinear model. After describing the dynamical model of the considered system, an adaptive gain sliding mode control (AGSMC) algorithm is proposed to regulate the concentration of some pollutants in a waste treatment process at a predefined constant (low) level. The stability is shown by the Lyapunov theory and the control action used did not exhibit any chattering behaviour. Several simulation results are provided to validate the proposed strategy and illustrate the overall performance improvements.
\end{abstract}

Keywords: Nonlinear systems, Biotechnological process, Adaptive gain sliding mode control, Stability.

\section{Introduction}

In the recent industry, the growth of advanced control algorithms for biotechnological processes is troubled by major difficulties $[1,8,14]$. These processes are toughly nonlinear and additionally the process parameters are highly uncertain. Another difficulty lies mostly in the lack, of low-cost and reliable measurement instrumentation.

Numerous methods have been proposed in the literature in order to overcome these difficulties such as adaptive approach [1,7] and artificial intelligence (AI)-based strategies such as fuzzy logic controller [4, 9], neural network [3, 11] and genetic algorithm [17]. The adaptive control provides a high performance for the bioprocess. Nevertheless, the occurrence of large and abrupt changes in the process parameters might lead to the failure of the control process. Furthermore, the application of AI-based strategies can lead to better performance of the bioprocess, and can offer good responses. However, the major problem of these strategies is the high computational burden, which will lead to increase the controllers cost.

During the last few years, the sliding mode control (SMC) algorithm has rapid expansion due to their advantages such as robustness, compact implementation, controller order reduction, low computational complexity, and insensitive to parameter changes. The SMC has been 
extensively accepted as an effective technique for the control of uncertain nonlinear systems $[15,18]$. Where the conventional SMC has been successfully applied in the electrical and mechanical process $[21,23]$. This strategy was recently extended to the chemical process [16]. Moreover, some attempts for the implementation of the SMC [2, 5, 20] and SMC combined with model-reference adaptive control laws [10, 13, 19] are suggested for bioprocess. These strategies offer good performance in the occurrence of parameter uncertainties and external disturbances.

For this purpose, an adaptation law is introduced in this paper to define the suitable switching gain in the discontinuous part of a conventional developed SMC controller for a nonlinear biotechnological process. Where the main contribution of this paper is to overcome both problems of perturbation rejection and chattering attenuation in sliding mode control strategy using adaptive control method. Thus, the proposed adaptive controller is composed of a classical SMC continuous part and a discontinuous part with an adaptive gain. The stability and convergence properties of this controller are proved through Lyapunov method. The main advantages of the use SMC algorithm with an adaptive gain is the enhancement of the system performances, mainly the system robustness and the settling time of the classical SMC technique. A complete simulation model for the proposed controller is developed with the MATLAB environment. Simulation results confirm the feasibility and performance improvement of the proposed controller at different operating conditions, compared to the conventional SMC.

\section{Dynamical model of the bioprocess}

In practice, the biotechnological process control is often limited to the regulation of the $\mathrm{pH}$ and temperature at constant values favourable to the microbial growth. There is, however, no doubt that the control of the biological state variables (biomass, substrates, etc.) can help to increase the performance. To develop and apply advanced control strategies for these biological variables, it is obligatory to obtain a useful dynamical model.

By means of a mass balance of the components inside the bioreactor and obeying some modelling rules, a dynamical state-space model of a prototype continuous bioprocess that takes place in a CSTB is defined by the following nonlinear system [1]:

$$
\left\{\begin{array}{l}
\dot{X}=\mu(S) X-D X \\
\dot{S}=-K_{1} \mu(S) X-D S+u, \\
e=X-C
\end{array}\right.
$$

where $X$ represents the biomass concentration, $[\mathrm{g} / \mathrm{l}] ; S$ is the substrate concentration, $[\mathrm{g} / \mathrm{l}]$; $D$ is the dilution rate, $\left[\mathrm{h}^{-1}\right] ; e$ is the error; $K_{1}$ is the yield coefficient. The control variable $u$ is defined as the substrate supply rate to the reactor per unit of volume, $[\mathrm{g} / \mathrm{l} \cdot \mathrm{h}]$, and $C$ represents the desired concentration of biomass, $[\mathrm{g} / \mathrm{l}]$. For this specific bioprocess, the reaction rate is of the form $\varphi(X, S)=\mu(S) X$, where $\mu(S)$ is the specific growth rate (a nonlinear function of $S$ ). There are numerous models for the specific growth rate, depending on the type of microorganisms involved in the reaction. One of the most common models for the specific growth rate is the Monod kinetic model:

$$
\mu(s)=\frac{\mu^{*} S}{\left(K_{M}+S\right)},
$$


where $\mu^{*}$ is the maximum specific growth rate and $K_{M}$ is the Michaelis-Menten constant.

The control goal for bioprocess is to control the concentration error $e$ to zero in order that the biomass concentration value $X$ should converge to a prescribed set-point value specified by the constant $C$.

\section{SMC and adaptive gain SMC controllers}

This section gives the detail of the SMC design of the bioprocess defined by the dynamical process (Eqs. (1) and (2)). In general, the SMC approach usually consists of two steps [18, 21]:

- Step 1: Sliding manifold design: define a fictitious output variable (referred to as the "sliding variable") depending on the measurable output and a certain number of its derivatives, whose vanishing guarantees that the resulting system behavior (i.e. the associated zero-dynamics) meets the desired performance specifications.

- Step 2: Controller design: define a control action that steers to zero in finite time the sliding variable, despite of model uncertainties and disturbances.

\section{SMC law}

As mentioned above, the control objective for the waste treatment processes is to control the concentration of some pollutants at a constant low level. So, one can consider the following auxiliary output function, which is called in this case the sliding surface:

$$
\sigma=\dot{e}+\lambda e=\mu(S) X-D X+\lambda(X-C),
$$

where $\sigma$ is the sliding surface and $\lambda$ is a positive constant.

It has a relative degree one with respect to the control input. Thus, one can use first order sliding mode control (FOSMC) algorithm to ensure the robust stabilization of the considered manifold in Eq. (3). The first time derivative of the output in Eq. (3) is given as:

$$
\begin{aligned}
\dot{\sigma} & =\left(\frac{\mu^{*} K_{M}}{\left(K_{M}+S\right)^{2}} \dot{S}\right) \mathrm{X}+\mu(S) \dot{X}-D \dot{X}+\lambda \dot{X}= \\
& =\mu^{\prime}(S) X\left(-K_{1} \mu(S) X-D S\right)+\mu^{\prime}(S) X u+(\mu(S)-D+\lambda)(\mu(S)-D),
\end{aligned}
$$

where

$$
\mu^{\prime}(S)=\frac{\mu^{*} K_{M}}{\left(K_{M}+S\right)^{2}}
$$

Eq. (4) can be expressed as follows:

$$
\dot{\sigma}=\Psi+\mu^{\prime}(S) X u=\Psi+\Gamma u=\Psi+d+\bar{u}
$$

with

$$
\left\{\begin{array}{l}
\Psi=\mu^{\prime}(S) X\left(-K_{1} \mu(S) X-D S\right)+(\mu(S)-D+\lambda)(\mu(S)-D), \\
\Gamma=\mu^{\prime}(S) X \text { and } \bar{u}=\Gamma u,
\end{array}\right.
$$


where the unknown function $d$ is introduced to represent all model uncertainties and external perturbations.

The functions $\Psi$ and $\Gamma$ are supposed to be such that:

$$
\left\{\begin{array}{l}
|\Psi| \leq \Psi_{M}, \\
0 \prec \Gamma_{m} \leq|\Gamma| \leq \Gamma_{M} .
\end{array}\right.
$$

It is assumed that $\Psi_{M}, \Gamma_{m}$ and $\Gamma_{M}$ exist but are unknown.

The best approximation of the control law that would achieve $\dot{\sigma}=0$ and ensures the robustness of the controlled system is:

$\bar{u}=\bar{u}_{e q}+\bar{u}_{s w}$

with

$\bar{u}_{e q}=-\Psi=\mu^{\prime}(S) X\left[K_{1} \mu(S) X+D S\right]-(\mu(S)-D+\lambda)(\mu(S)-D) X$

and

$\bar{u}_{s w}=-K \operatorname{sgn} \sigma, K \succ|d|$,

where $K$ is a strictly positive scalar.

The control input $u$ in such case may be defined as:

$$
\begin{aligned}
u & =\frac{\bar{u}}{\mu^{\prime}(S) X}=K_{1} \mu(S) X-\frac{(\mu(S)-D)^{2}}{\mu^{\prime}(S)}- \\
& -\frac{1}{\mu^{\prime}(S) X}(\lambda(\mu(S)-D) X+K \operatorname{sgn}(\lambda(X-C)+(\mu(S)-D) X)) .
\end{aligned}
$$

\section{Adaptive SMC law}

The performances of the above discussed SMC algorithm depends on the suitable selection of the gain $K$. Due to the high level of nonlinearity in the considered system and the presence of external disturbances, we can choose to increase the switching gain $K$ to overcome the precise stabilization and the perturbation rejection problems in control design. However, higher coefficient may produce a larger chattering phenomenon, with an amplitude proportional to the discontinuity magnitude in the controller. Thus, the overall controller is composed of continuous part, as in Eq. (9), and a discontinuous part with an adaptive gain.

The gain $K$ is adapted to the sliding mode output according to the following algorithm:

$$
K(t)= \begin{cases}\int_{0}^{t} \bar{K}_{1} \cdot|\sigma(x, t)| d t & \text { if }|\sigma(x, t)| \succ \varepsilon \succ 0, \\ \bar{K}_{2} \cdot|\eta|+\bar{K}_{3} & \text { if }|\sigma(x, t)| \leq \varepsilon,\end{cases}
$$


where

$$
\tau \dot{\eta}+\eta=\operatorname{sgn}(\sigma(x, t))
$$

and $\bar{K}_{1}, \bar{K}_{2}$ and $\bar{K}_{3}$ are constants and vary when there is a change in the surface sign at time $t^{*}$. If $t^{*}$ is the largest time value and $t^{*_{-}}$denotes the time just before $t^{*}, \sigma\left(x\left(t^{*_{-}}\right), t^{*_{-}}\right) \succ \varepsilon$ and $\sigma\left(x\left(t^{*}\right), t^{*}\right) \leq \varepsilon$ for a suitable positive parameter $\varepsilon$.

\section{Stability analysis}

We start with the study of stability of the classical SMC and then the study of stability of the adaptive SMC.

The stability of the system under the control input (Eq. (12)) may be shown by choosing the following Lyapunov function that is positive definite:

$$
V=\frac{1}{2} \sigma^{2} \succ 0 \text {. }
$$

First time derivative is as follows:

$\dot{V}=\sigma \dot{\sigma}$

Introducing $u$ in Eq. (16), one can get:

$\dot{V}=\sigma\left(\left(\mu^{\prime}(S)\left(-K_{1} \mu(S) X-D S+u\right)\right) X+(\mu(S)-D+\lambda)(\mu(S)-D) X\right)$,

then

$\dot{V}=\sigma(-K \operatorname{sgn} \sigma)=-K|\sigma| \prec 0$

which implies the stability of the studied system.

Remark 1: The Singularity problem may be faced in the control design when $X=0$ is devoid of practical significance ( $X=0$ means no microorganisms and the life in the bioreactor is stopped).

We go to study the stability in the case of adaptive gain SMC (AGSMC):

- First case: $|\sigma(x, t)| \succ \varepsilon \succ 0$

Consider the following Lyapunov candidate function, where $K^{*}$ is a positive constant:

$V=\frac{1}{2} \sigma^{2}+\frac{1}{2 \gamma}\left(K-K^{*}\right)^{2}$

and 
$\dot{V}=\sigma \Psi-\sigma \Gamma \mathrm{K} \operatorname{sign}(\sigma)+\frac{1}{\gamma}\left(K-K^{*}\right) \bar{K}_{1}|\sigma|$

Therefore

$$
\begin{aligned}
& \dot{V} \leq \Psi_{M}|\sigma|-\Gamma_{m} \mathrm{~K}|\sigma|+\frac{1}{\gamma}\left(K-K^{*}\right) \bar{K}_{1}|\sigma|, \\
& \dot{V} \leq \Psi_{M}|\sigma|-\Gamma_{m} \mathrm{~K}|\sigma|+\Gamma_{m} \mathrm{~K}^{*}|\sigma|-\Gamma_{m} \mathrm{~K}^{*}|\sigma|+\frac{1}{\gamma}\left(K-K^{*}\right) \bar{K}_{1}|\sigma|, \\
& \dot{V} \leq\left(\Psi_{M}-\Gamma_{m} \mathrm{~K}^{*}\right)|\sigma|+\left(K-K^{*}\right)\left(-\Gamma_{m}|\sigma|+\frac{\bar{K}}{\gamma}|\sigma|\right) .
\end{aligned}
$$

There always exists $K^{*} \succ 0$ such that $K-K^{*} \prec 0$ for all $t \succ 0$. It yields:

$$
\begin{aligned}
\dot{V} & =-\left(-\Psi_{M}+\Gamma_{m} \mathrm{~K}^{*}\right)|\sigma|-\left(-\Gamma_{m}|\sigma|+\frac{\bar{K}}{\gamma}|\sigma|\right)\left|K-K^{*}\right| \\
& =-\beta_{\sigma}|\sigma|-\beta_{K}\left|K-K^{*}\right|
\end{aligned}
$$

with $\beta_{\sigma}=-\Psi_{M}+\Gamma_{m} \mathrm{~K}^{*}$ and $\beta_{K}=-\Gamma_{m}|\sigma|+\frac{\bar{K}}{\gamma}|\sigma|$.

There always exists $K^{*}$ and $\gamma$ such that $K^{*} \succ \frac{\bar{K}}{\Gamma_{m}}$ and $\gamma \prec \frac{\bar{K}}{\Gamma_{m}}$, which yields $\beta_{\sigma} \succ 0$ and $\beta_{k} \succ 0$. Then, we get:

$$
\begin{aligned}
& \dot{V}=-\beta_{\sigma} \sqrt{2} \frac{|\sigma|}{\sqrt{2}}-\beta_{K} \sqrt{2 \gamma} \frac{\left|K-K^{*}\right|}{\sqrt{2 \gamma}} \\
& \dot{V} \leq-\min \left\{\beta_{\sigma} \sqrt{2}, \beta_{K} \sqrt{2 \gamma}\right\}\left(\frac{|\sigma|}{\sqrt{2 \gamma}}+\frac{\left|K-K^{*}\right|}{\sqrt{2 \gamma}}\right) \leq-\beta V^{1 / 2}
\end{aligned}
$$

with: $\beta=\min \left\{\beta_{\sigma} \sqrt{2}, \beta_{K} \sqrt{2 \gamma}\right\}$.

- Second case: $|\sigma(x, t)| \leq \varepsilon$

According to the developed theorem in [6], if $K$ is large enough with respect to perturbations/uncertainties effects, then the proposed sliding mode control with the gain adaptation algorithm Eq. (13) allows maintaining responses of system on the sliding surface $\sigma=0$. Assumed that responses of system reach $\sigma=0$, it means that $K$ is large enough as required by theorem in [12]. Before, the sliding mode is established in system for all $t \geq t_{r}$ (where $t_{r}$ represents the reaching time of the sliding control). Therefore, the theorem is confirmed. From Eqs. (8) and (9), we can note that the main feature of this approach is that it does not require a priori the knowledge of control gain. The auto-tuning of the control parameter consists in increasing the control gain $K$, from an initial value, since a sliding mode is established. 


\section{Results and discussion}

The simulated control tasks considers the problem of stabilizing the output $e$ to zero, which is equivalent to the biomass concentration $X$ converging to a pre-specified constant reference value $C$, where the values considered for the process parameters [12] are $K_{1}=12, \mathrm{D}=0.2 \mathrm{~h}^{-1}$, $\mu^{*}=2.1 \mathrm{~h}^{-1}, K_{M}=10 \mathrm{~g} / \mathrm{l}$. In order to analyse the behaviour and to test the performance of the controllers, the following simulation cases are considered:

- The SMC law Eq. (12) is implemented for the continuous bioprocess, with the control parameters $K=3, \lambda=1$, and the set-point for the biomass concentration $C=50 \mathrm{~g} / \mathrm{l}$. A step change in the set-point is provided from the initial value $C=50 \mathrm{~g} / \mathrm{l}$ to the value $C=35 \mathrm{~g} / 1$.

- Control input $u$ and gain adaptation law $K(t)$ (Eqs. (12) and (13)). Gain is initialized at $K(0)=10$, its dynamics being tuned with $\bar{K}_{1}=10, \bar{K}_{3}=1$ and $\tau=3 \mathrm{~s}$.

\section{Test 1: Nominal case}

We consider the nominal case where the system model is known and no external disturbance signal affects the system. Figs. 1 and 2 depict the evolution of biomass concentration and the tracking error, respectively, of the nominal process under the above-discussed controllers.

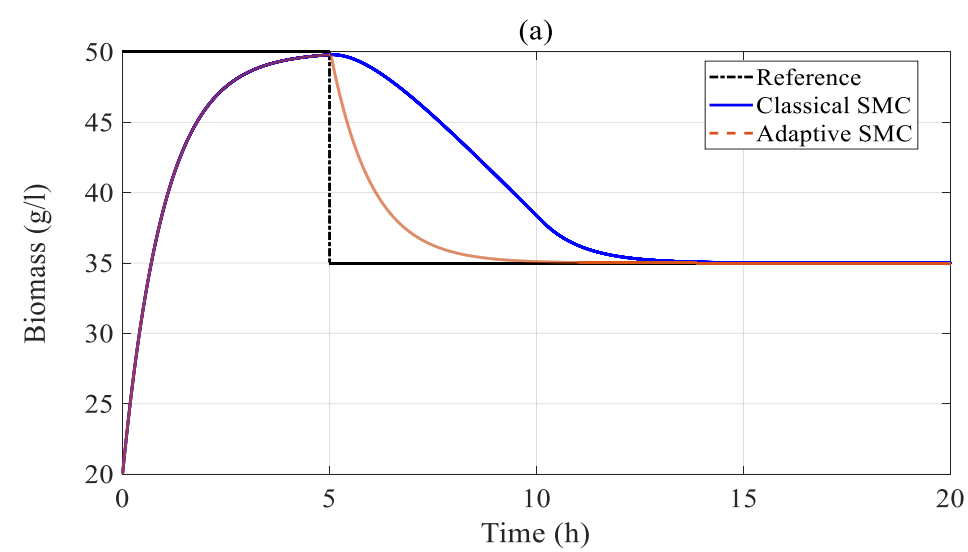

(b)

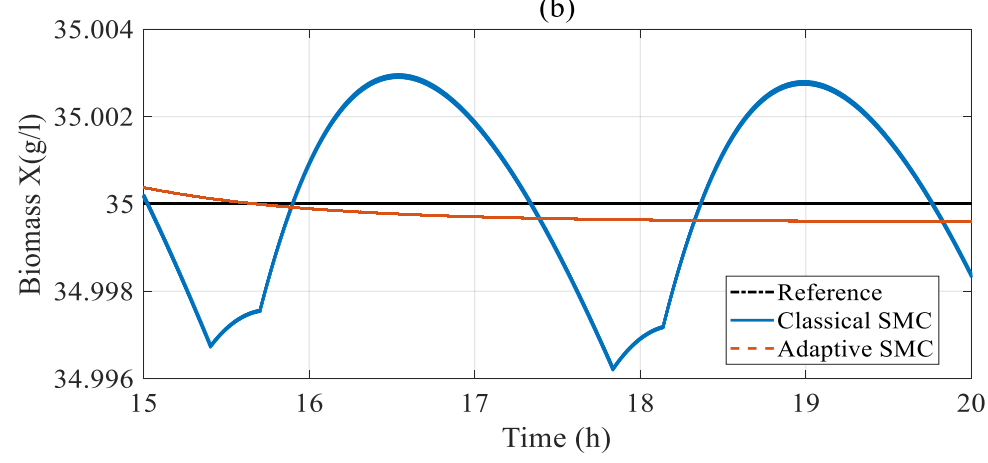

Fig. 1 a) Time response of biomass concentration with SMC and AGSMC;

b) zoom view.

Compared to the classical SMC, it is clear that the proposed algorithm allows achieving fast convergence of system to its desired reference in Fig. 1a and Fig. 2. Moreover, it is clearly seen from Fig. 1b that the AGSMC allows to attenuate the chattering phenomena characterizes the response of the system under a fixed gain SMC. 


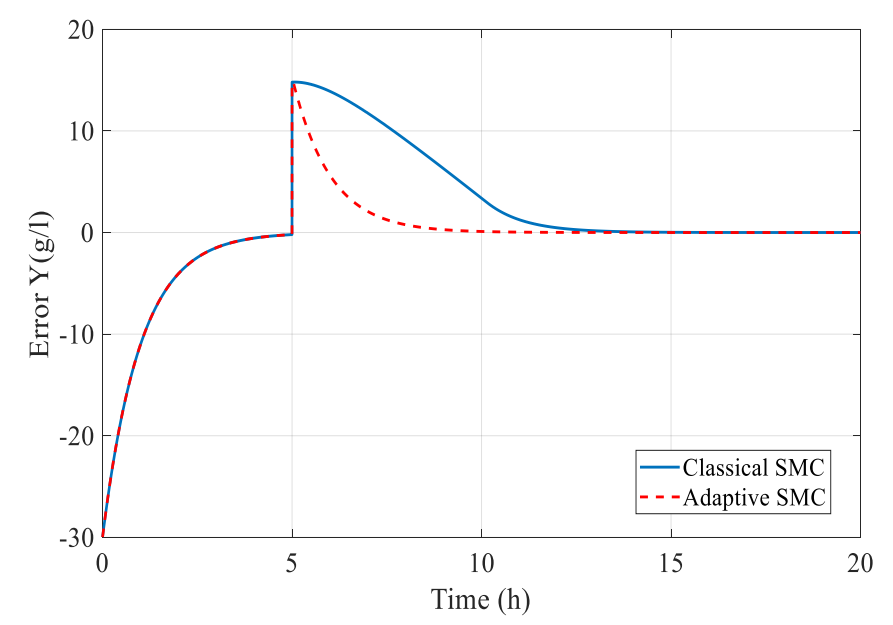

Fig. 2 Error evolution of biomass with SMC and AGSMC

For the final value of substrate concentration $S$, the observed chattering phenomenon is always greater in the classical SM control as shown in Fig. 3. The final value of $S$ is almost constant from $13 \mathrm{~h}$ and reaches a constant value of $1.05 \mathrm{~g} / \mathrm{l}$ at $14 \mathrm{~h}$ in the proposed control (AGMSC). On the other hand, for the classical control (SMC), the reaching time of the final value is more than $19 \mathrm{~h}$.

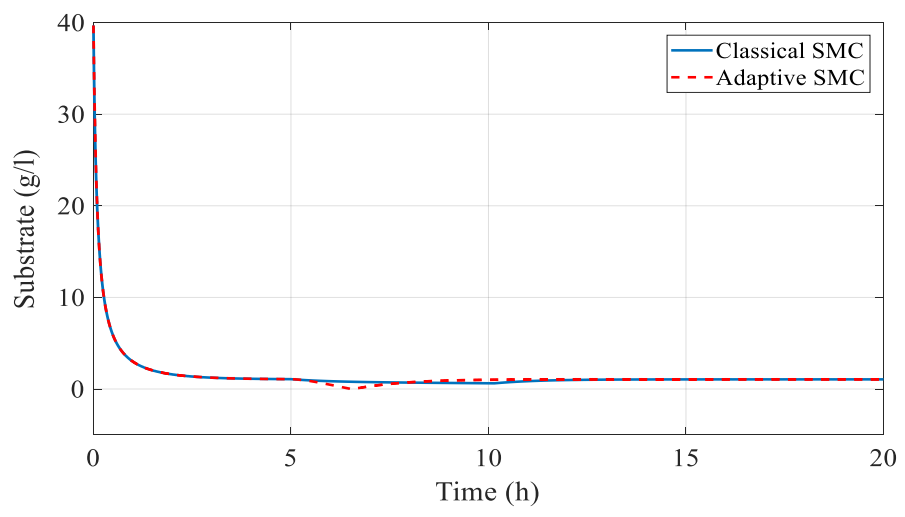

a)

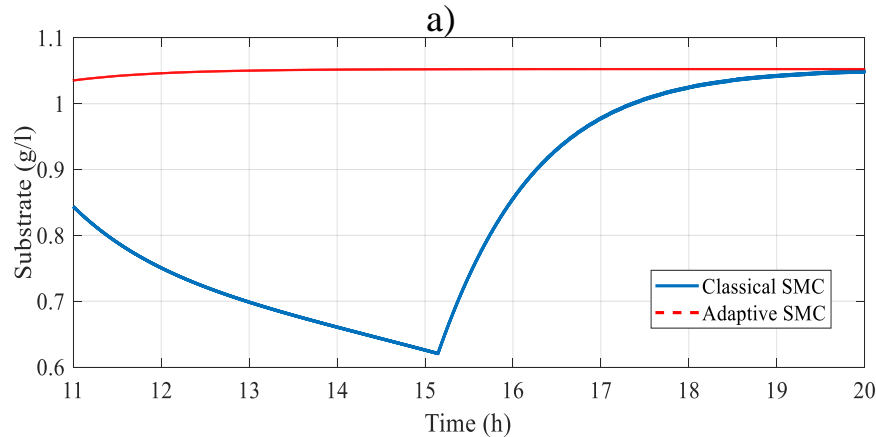

b)

Fig. 3 a) Time evolution of substrate concentration with SMC and AGSMC, b) zoom view.

As for the substrate concentration $S$, the non-trivial equilibrium point $S_{e}$ is given by [22]:

$$
S_{e}=\frac{K_{M} D}{\mu^{*}-D}=1.05
$$


Fig. 4a highlights the evolution of the control signals in both cases. The final value of $u$ is more oscillatory in the classical control law (SMC) and the proposed one (AGSMC) (Fig. 4b).

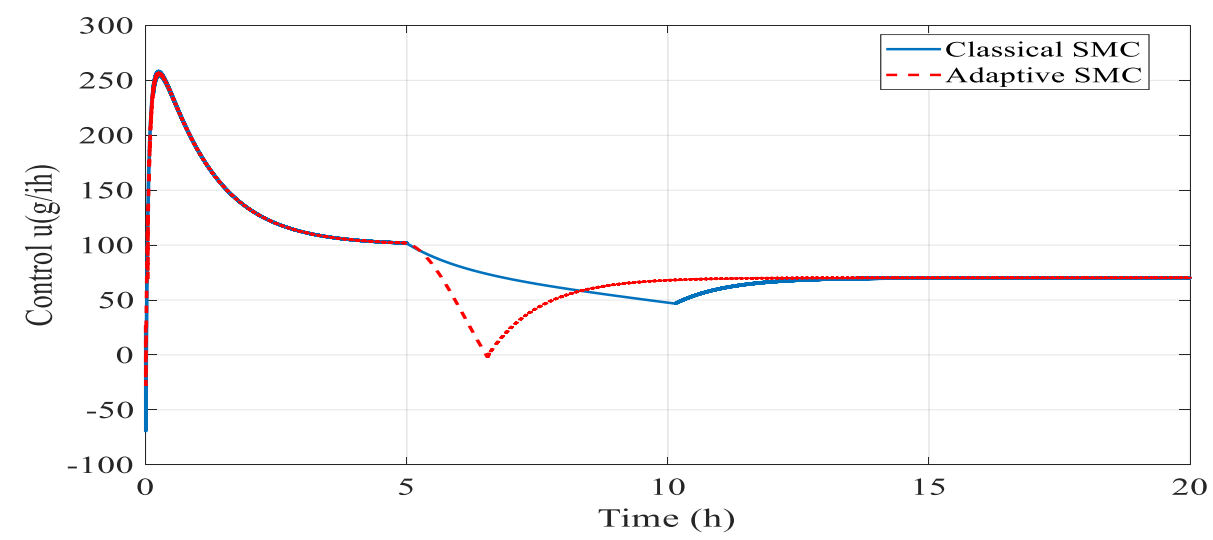

a)

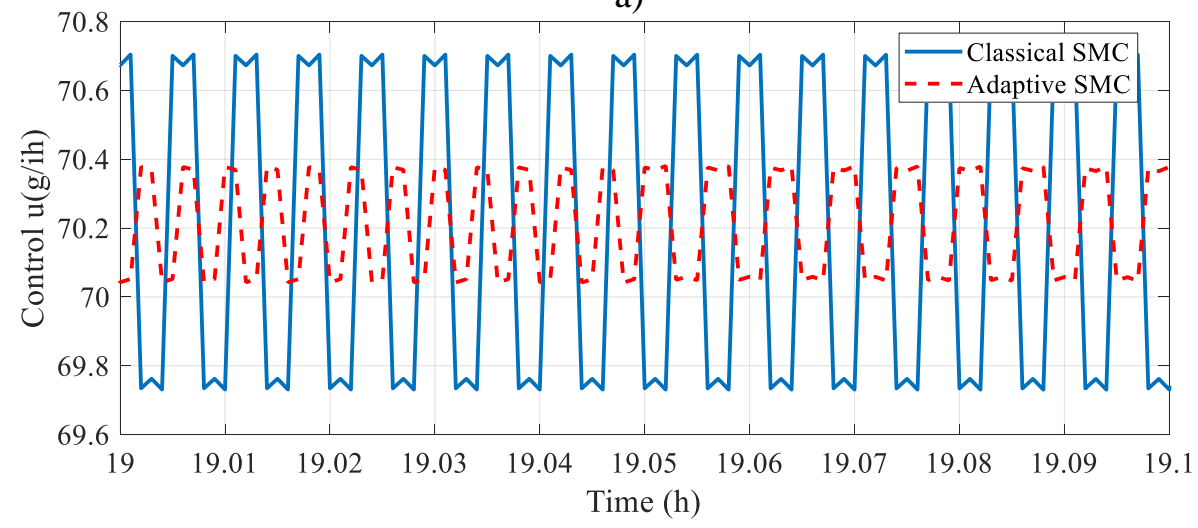

b)

Fig. 4 a) Control action of the SMC and AGSMC; b) zoom view.

The gain parameter variation (AGSMC) is shown in Fig. 5. In order to compensate quickly the abrupt change in the desired concentration of the biomass, in the time, an increased value of the gain is established. Moreover, when the biomass concentration reaches its desired value, the parameter gain decreases to a low value. The value gain proposed in [12] $(K=3)$ has been used for the classical control, meanwhile with adaptive control, as shown in Fig. 5, the value of this gain is generally equal to 1 .

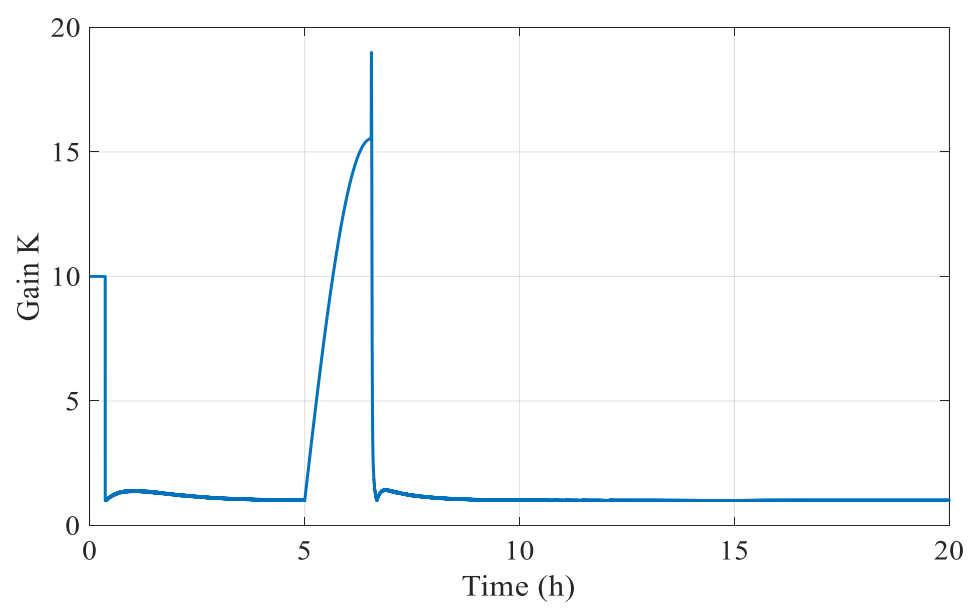

Fig. 5 Variation of the controller AGSMC gain 


\section{Test 2: Perturbed case}

In order to evaluate the robustness of the proposed control algorithm (AGSMC) and to compare with the robustness of the classical control law (SMC), the system was considered in a second test to be subjected to a randomly distributed external disturbance of a maximum value 0.072 , i.e. we add a noise at the first equation:

$$
\dot{X}=\mu(S) X-D X+W
$$

with $W$ is an external disturbance.

Fig. 6 represents a comparative study between the nominal case and the perturbed one for system evolution under the classical (SMC) algorithm, where the noise has an effect on the biomass concentration response. The system evolution in both cases under the proposed (AGSMC) algorithm is shown in Fig. 7. As seen, the system performances are preserved under the proposed control approach in both cases.

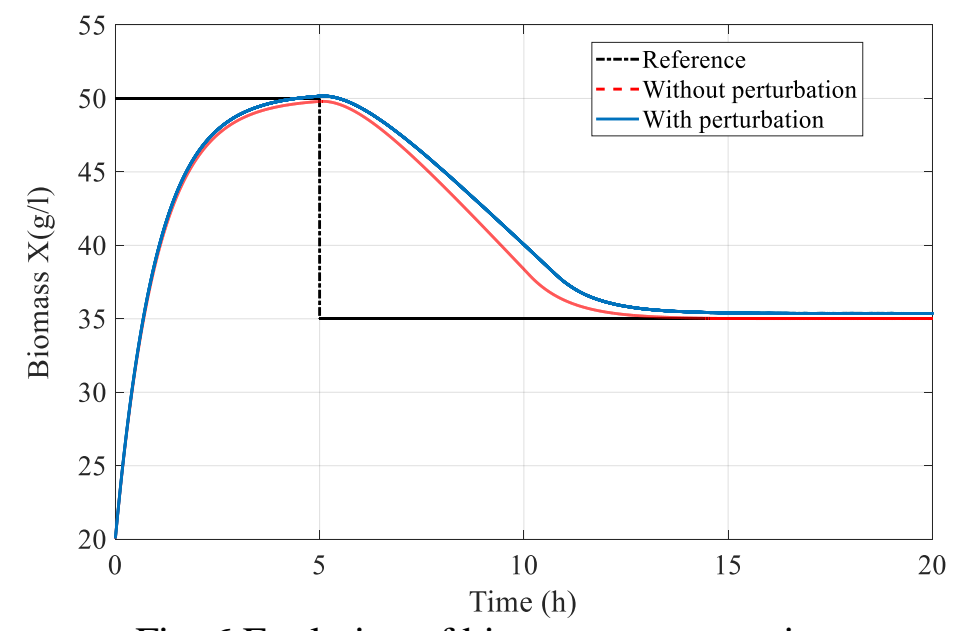

Fig. 6 Evolution of biomass concentration under SMC in perturbed and unperturbed case

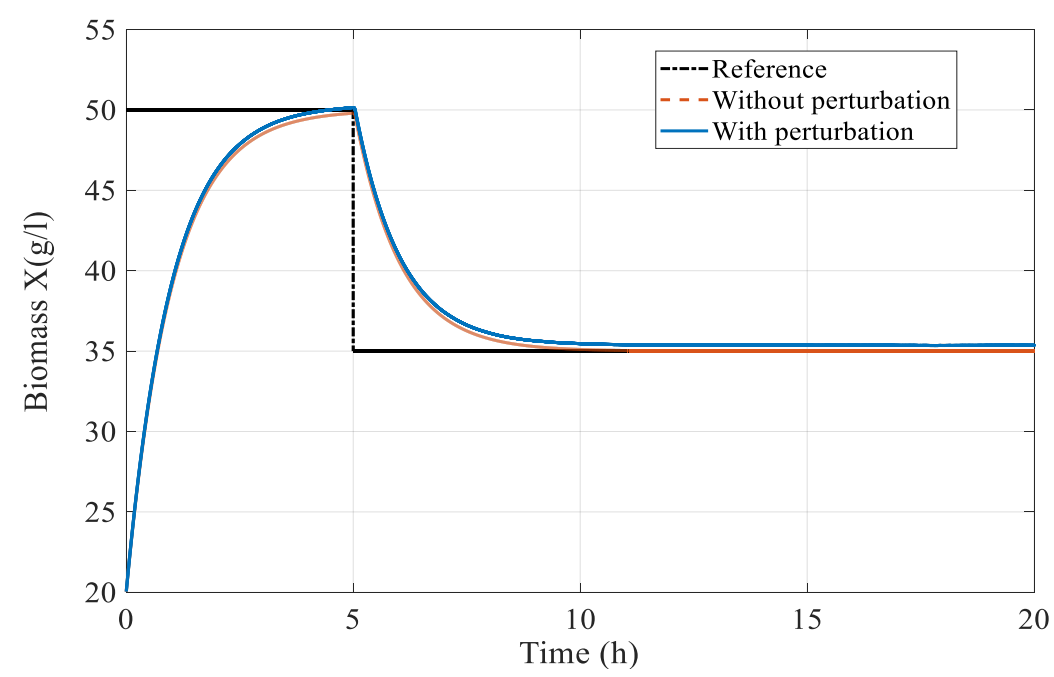

Fig. 7 Evolution of biomass concentration under AGSMC in perturbed and unperturbed case 
Fig. 8 represents the time evolution of the substrate under the classical SMC and the proposed AGSMC in perturbation case. Concentration of substrate reaches the final value in the case AGSMC (at $17 \mathrm{~h}$ ) before the case SMC (after $19 \mathrm{~h}$ ). Fig. 9 depicts the variation of the controller gains in the perturbed and unperturbed cases. In the AGSMC control, it varies and takes into account the presence of noise. In the classical SMC control, $K$ is constant. Fig. 10 shows the control action of the SMC and AGSMC in perturbed case. The oscillation band is narrower with AGSMC control compared to the classical SMC control.

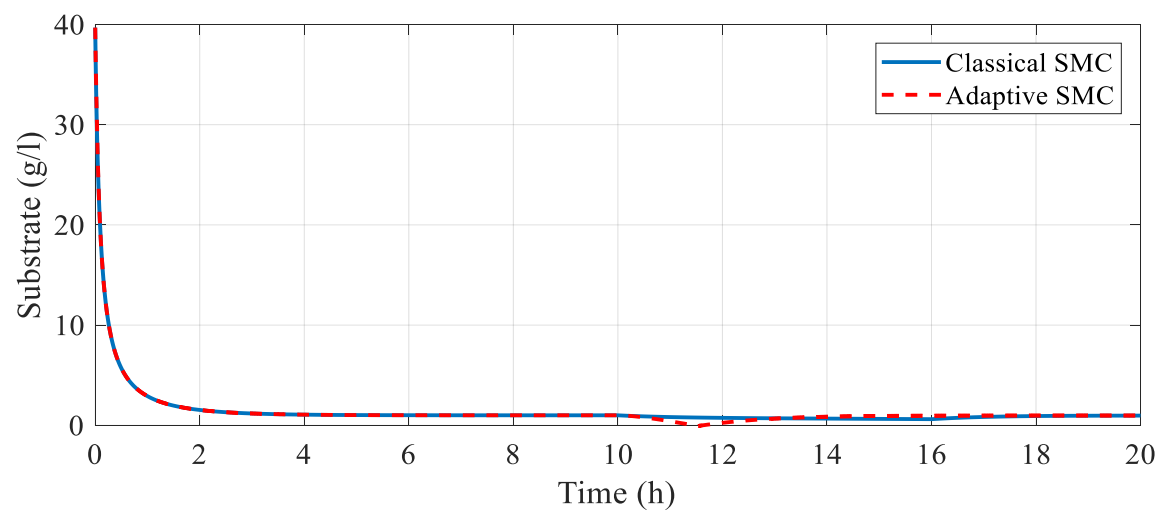

a)

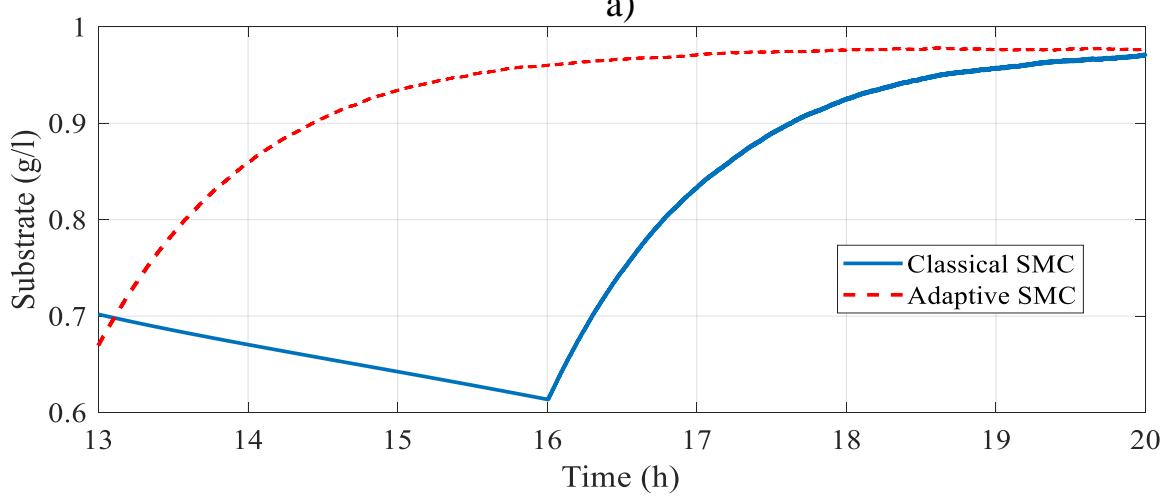

b)

Fig. 8 a) Evolution of substrate concentration with SMC and ASMC in the perturbation case;

b) zoom view.

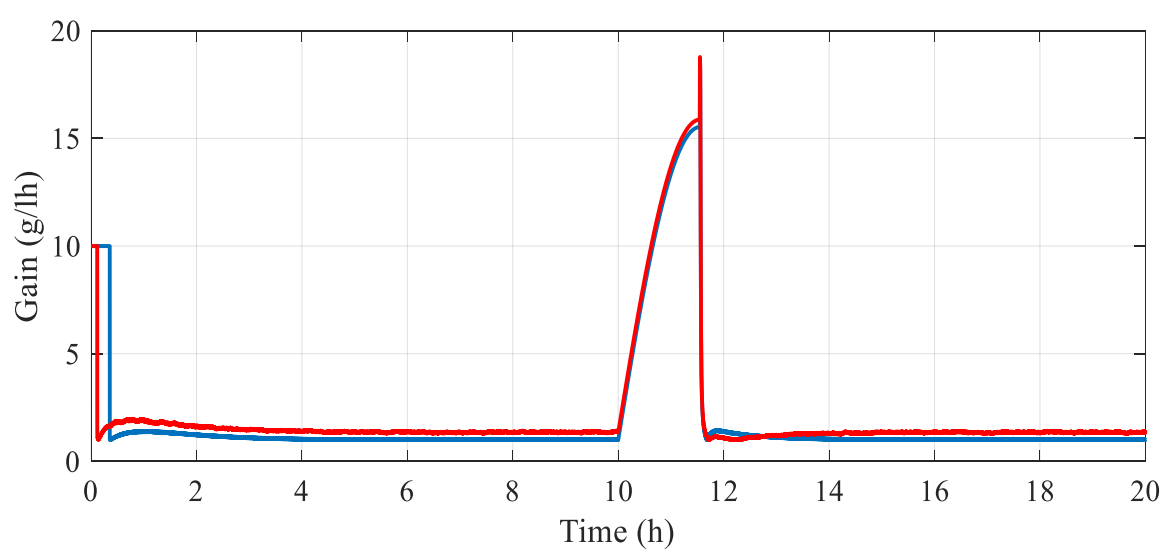

Fig. 9 Variation of the controller AGSMC gain in perturbed and unperturbed case 


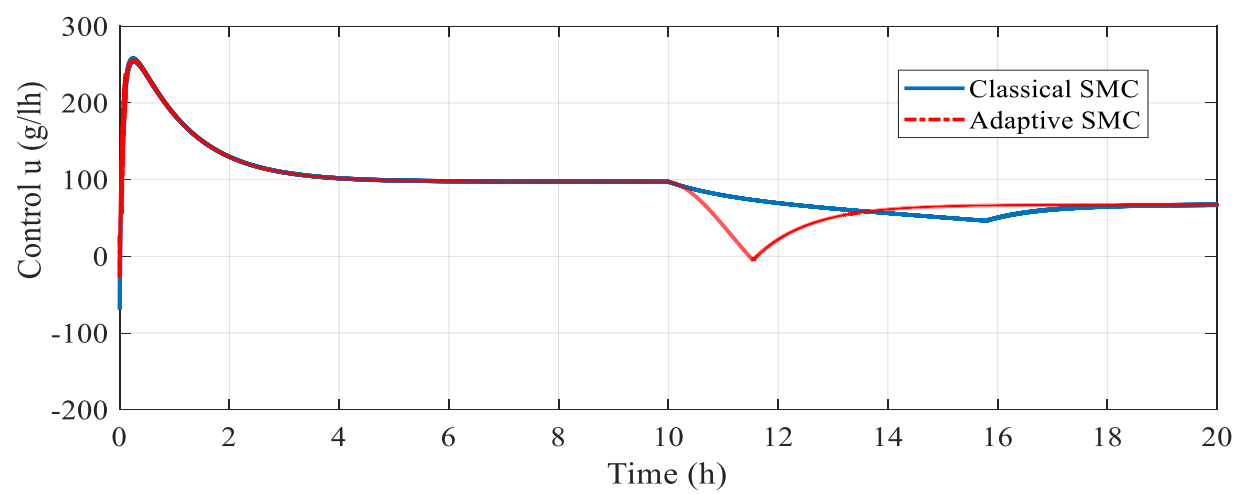

a)

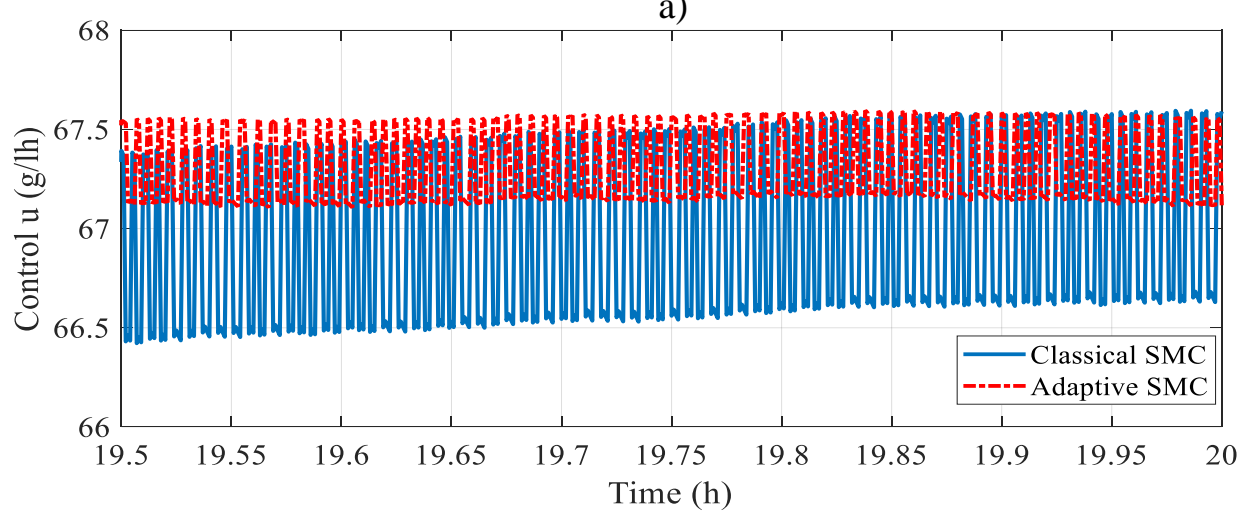

b)

Fig. 10 a) Control action of the SMC and AGSMC in perturbed case;

b) zoom view.

\section{Conclusion}

In this paper an adaptive gain sliding mode controller has been developed for the accurate stabilization of a nonlinear biotechnological process. Firstly, a classical sliding mode control algorithm is developed. Then the appropriate control parameters are obtained through a dynamical adaptation of switching gain to obtain fast convergence of system to its desired value. Simulation results are presented to illustrate the effectiveness of the proposed approach. Compared to the classical sliding mode control techniques, AGSMC method performs better than classical SMC method in terms of the stabilization accuracy, and system rapidity and robustness.

\section{References}

1. Bastin G., D. Dochain (1999). On-line Estimation and Adaptive Control of Bioreactors, New York, Elsevier.

2. Fossas E., R. M. Ros, J. Fabregat (2001). Sliding Mode Control in a Bioreactor Model, Journal of Mathematical Chemistry, 30(2), 203-218.

3. Gadkar K. G., S. Mehra, J. Gomes (2005). On-line Adaptation of Neural Networks for Bioprocess Control, Computers \& Chemical Engineering, 29(5), 1047-1057.

4. Honda H., T. Kobayashi (2000). Fuzzy Control of Bioprocess in Japan, Biotechnology Annual Review, 5, 1-23.

5. Hristozov I., T. Pencheva, D. Huell, C. Klockow, B. Hitzmann, S. Tzonkov (2005). Implementation of Sliding Mode Controller with Boundary Layer for Saccharomyces cerevisiae Fed-batch Cultivation, International Journal Bioautomation, 2, 49-53. 
6. Lee H., V. I. Utkin (2007). Chattering Suppression Methods in Sliding Mode Control Systems, Annual Reviews in Control, 31(2), 179-188.

7. Mailleret L., O. Bernard, J.-P. Steyer (2004). Nonlinear Adaptive Control for Bioreactors with Unknown Kinetics, Automatica, 40(8), 1379-1385.

8. Neria-González M. I., R. Aguilar-López (2015). Uncertainty Estimator Based Nonlinear Feedback Control for Tracking Trajectories in a Class of Continuous Bioreactor, International Journal Bioautomation, 19(1), 43-60.

9. Nyttle V. G., M. Chidambaram (1993). Fuzzy Logic Control of a Fed-batch Fermentor, Bioprocess Engineering, 9(2-3), 115-118.

10. Plestan F., Y. Shtessel, V. Brégeault, A. Poznyak (2010). New Methodologies for Adaptive Sliding Mode Control, International Journal of Control, 83(9), 1907-1919.

11. Schmitt F., R. Banu, I.-T. Yeom, K.-U. Do (2018). Development of Artificial Neural Networks to Predict Membrane Fouling in an Anoxic-aerobic Membrane Bioreactor Treating Domestic Wastewater, Biochemical Engineering Journal, 133, 47-58.

12. Selişteanu D., E. Petre, V. B. Răsvan (2007). Sliding Mode and Adaptive Sliding-mode Control of a Class of Nonlinear Bioprocesses, International Journal of Adaptive Control and Signal Processing, 21(8-9), 795-822.

13. Shtessel Y. B., J. A. Moreno, L. M. Fridman (2017). Twisting Sliding Mode Control with Adaptation: Lyapunov Design, Methodology and Application, Automatica, 75, 229-235.

14. Simeonov I., B. Kalchev, S. Mihaylova, V. Hubenov, A. Aleksandrov, R. Georgiev, N. Christov (2012). Pilot-scale Biogas Plant for the Research and Development of New Technologies, International Journal Bioautomation, 16(3), 187-202.

15. Sira-Ramírez H. (1992). On the Sliding Mode Control of Nonlinear Systems, Systems \& Control Letters, 19(4), 303-312.

16. Sira-Ramirez H., O. Llanes-Santiago (1994). Dynamical Discontinuous Feedback Strategies in the Regulation of Nonlinear Chemical Processes, IEEE Transactions on Control Systems Technology, 2(1), 11-21.

17. Slavov T., O. Roeva (2011). Genetic Algorithm Tuning of PID Controller in Smith Predictor for Glucose Concentration Control, International Journal Bioautomation, 15(2), 101-114.

18. Slotine J. J., W. Li (1991). Applied Nonlinear Control, New Jersey, Prentice-Hall: Englewood Cliffs.

19. Stanchev S. P. (2003). A Variant of an (Combined) Adaptive Controller Design Introducing Sliding Regime in Lyapunov Derivative, Proceedings of the 2003 American Control Conference, doi: 10.1109/ACC.2003.1239139.

20. Tham H. J., K. B. Ramachandran, M. A. Hussain (2003). Sliding Mode Control for a Continuous Bioreactor, Chemical and Biochemical Engineering, 17(4), 267-275.

21. Utkin V., J. Guldner, J. Shi (2009). Sliding Mode Control in Electromechanical Systems, Boca Raton, Taylor \& Francis.

22. Wang H. P., B. Kalchev, Y. Tian, I. Simeonov, N. Christov, C. Vasseur (2011). Composed Adaptive Control for a Second-order Nonlinear Model of a Biotechnological Process, Proceedings of $19^{\text {th }}$ Mediterranean Conference on Control Automation (MED), doi: 10.1109/MED.2011.5983138.

23. Young K. D., V. I. Utkin, U. Ozguner (1999). A Control Engineer's Guide to Sliding Mode Control, IEEE Transactions on Control Systems Technology, 7(3), 328-342. 


\section{Semcheddine Bouyahia, Ph.D. Student}

E-mail: adelbouyahia@gmail.com

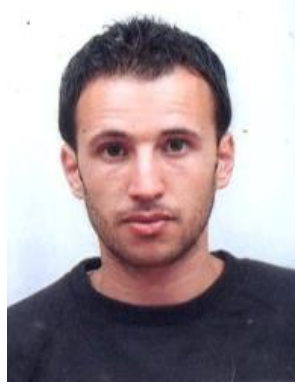

Semcheddine Bouyahia is a Ph.D. student in the Department of Electronics at the University of Ferhat Abbas Sétif 1, Sétif, Algeria where he obtained Master and Engineering degree in Electronics. He has been a college teacher since 2016. His research interests include control and biotechnological processes. He communicated several papers already.

\section{Prof. Samia Semcheddine, Ph.D. \\ E-mail: tssamia@yahoo.fr}

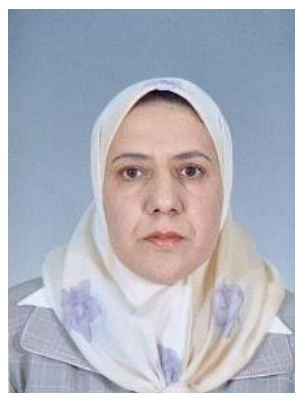

Samia Semcheddine was born on November 26, 1968 in Sétif, Algeria. She graduated from University of Ferhat Abbas Sétif 1, Sétif, Algeria in 2007. She became an Assistant Professor in the Department of Electronics at the University of Ferhat Abbas Sétif 1, Algeria in 1998. She is currently a Full Professor in Department of Electronics where she teaches course of automatic materials. Her research focuses on control and biotechnology. Recently, she is interested in renewable energy and, particularly, in biogas.

\section{Billel Talbi, Ph.D.}

E-mail: bilel_ei@live.fr

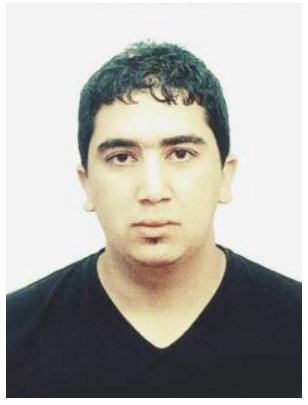

Billel Talbi received his B.Tech., M.Sc., and Ph.D. degrees in Electronics Engineering from the University of Ferhat Abbas Sétif 1, Sétif, Algeria, in 2011, 2013, and 2018, respectively. He is a member of the Laboratory of Power Electronics and Industrial Electronics, University of Ferhat Abbas Sétif 1. His research interests include control of power converters, power conversion techniques, and renewable energy systems.

\section{Oussama Boutalbi, Ph.D. Student}

E-mail: botalbioussama@gmail.com

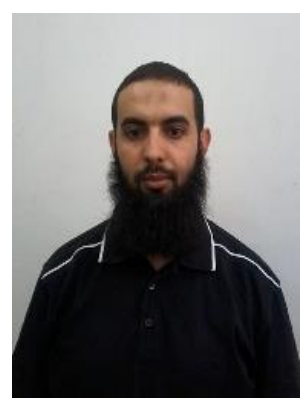

Oussama Boutalbi received his Engineer and Master degrees from University of Ferhat Abbas Sétif 1, in 2010 and 2014, respectively. Since 2013, he is with Intelligent Systems Laboratory (LSI), University of Ferhat Abbas Sétif 1. His research interests are motion planning, tracking control design of autonomous robot systems and robust control design for nonlinear systems.

(C) 2020 by the authors. Licensee Institute of Biophysics and Biomedical Engineering, Bulgarian Academy of Sciences. This article is an open access article distributed under the terms and conditions of the Creative Commons Attribution (CC BY) license (http://creativecommons.org/licenses/by/4.0/). 